\title{
¿Dependencia bicéfala o autonomía? América Latina en el espacio de la movilidad estudiantil internacional
}

Víctor Montoya

\section{RESUMEN}

Se propone en este artículo analizar la movilidad estudiantil internacional latinoamericana a partir de un marco teórico que articula la teoría de la dependencia y el concepto de campo de Bourdieu. Se realiza un análisis factorial de la correspondencia de la movilidad estudiantil internacional para describir ese espacio y luego se ubica y se detalla la posición de América Latina. El principal resultado es que la región constituye un polo relativamente autónomo que articula movilidades Norte-Sur-Sur, sin embargo, la región se encuentra en una relación de dependencia bicéfala con los países anglosajones y Europa.

Palabras clave: movilidad de estudiantes, internacionalización, análisis estadístico, educación superior, regionalización.

\section{Víctor Montoya}

vmontoya@mail.utdt.edu

Argentino-francés. Master en Políticas Comparadas, Institut d'Études Politiques de Aix-en-Provence, Francia; Candidato a Doctor en Estudios Internacionales, Universidad Torcuato Di Tella, Argentina; Doctorando en Ciencias Políticas, Centre de Recherche et de Docuementation sur les Amériques (IHEAL-CREDA), Université Sorbonne Nouvelle-Paris 3, Francia. Temas de investigación: internacionalización de la educación superior, política exterior, cooperación internacional, sociología política internacional. ORCID: 0000-0003-3890-8834 


\section{Dependência bicéfala ou autonomia? América Latina no espaço da mobilidade estudantil internacional}

\section{RESUMO}

Se propõe neste artigo analisar a mobilidade estudantil internacional latino-americana a partir de um marco teórico que articula a teoria da dependência e o conceito de campo de Bourdieu. Se realiza uma análise fatorial das correspondências da mobilidade estudantil internacional para descrever esse espaço e logo se localiza e se detalha a posição da América Latina. O principal resultado é que a região constitui um polo relativamente autônomo que articula mobilidades Norte-Sul-Sul, porém, a região se encontra em uma relação de dependência bicéfala com os países anglo-saxões e Europa.

Palavras chave: mobilidade de estudantes, internacionalização, análise estatístico, educação superior, regionalização.

Double-headed dependence or autonomy? Latin America in the space of international student mobility

\section{ABSTRACT}

This article proposes to analyze the Latin American international student mobility based on a theoretical framework that articulates the theory of dependence and Bourdieu's concept of "field". A factorial analysis of the correspondence of international student mobility is carried out to describe this space and then the position of Latin America is located and detailed. The main result is that the region constitutes a relatively autonomous pole that articulates North-South-South mobilities; however, the region is in a two-headed dependency relationship with Anglo-Saxon countries and Europe.

Key words: student mobility, internationalization, statistical analysis, higher education, regionalization. 


\section{Introducción}

El objetivo general de este artículo es analizar, a partir del estudio estadístico de la movilidad estudiantil internacional, la posición de América latina y el Caribe dentro del campo universitario transnacional y discutir su carácter periférico y dependiente. El segundo objetivo es completar los resultados obtenidos en otras investigaciones sobre los flujos estudiantiles internacionales, haciendo hincapié en el estudio "La movilidad en la educación superior en América Latina y el Caribe: retos y oportunidades de un convenio renovado para el reconocimiento de estudios, títulos y diplomas" publicado por el Instituto para la Educación Superior en América latina y el Caribe (IESALC, 2019). El enfoque propuesto se basa en la teoría de la dependencia, el concepto de campo de Bourdieu y se apoya en el Análisis Factorial de las Correspondencias para describir la estructura de la movilidad estudiantil. Se establecerá en primer lugar el marco conceptual y se explicará la metodología. Luego se hará la topografía del espacio de la movilidad internacional y se propondrá una interpretación de la estructura de ese espacio. En tercer lugar, se detallará el caso de América Latina estudiando la movilidad saliente y entrante.

\section{Marco conceptual y metodología}

El estudio de la dimensión universitaria de las relaciones internacionales y de la dimensión internacional de las prácticas universitarias da lugar al uso y a la formulación de una serie de conceptos y de teorías, como el de internacionalización, que Knight (2013) distingue por ejemplo de internacional y de global, o los de internacionalización en casa y en el exterior (Beelen y Jones, 2015). En cuanto al estudio específico de la movilidad estudiantil internacional, tal como lo indica Shields, existen al menos tres enfoques teóricos generales (neoliberal, crítico y neoinstitucional) que permiten abordajes diferentes y a partir de los cuales se pueden formular hipótesis diferentes (Shields, 2013). Ferrari-Martínez muestra además que el análisis de los flujos de estudiantes moviliza tres "constructos geográficos operantes en imaginarios de internacionalización": globalización, límite/frontera y escala geográfica (Ferrari-Martínez, 2019: 180). El planteo que hace Shields invita a revisar críticamente los marcos teóricos que se usan para analizar la movilidad estudiantil internacional, dado que éstos determinan las hipótesis que se formulan y por lo tanto los hallazgos que se pueden hacer. En cuanto al planteo que hace Ferrari-Martínez sobre los constructos geográficos, implica revisar críticamente las categorías geográficas que se utilizan para analizar la movilidad estudiantil internacional dado que estás tienen por definición una dimensión geográfica. Se desprende de las breves consideraciones anteriores la importancia de clarificar el marco conceptual y la metodología. Por lo tanto, se procederá en primer lugar a exponer ese marco para luego presentar las características y la relevancia del Análisis Factorial de las Correspondencias como método estadístico para estudiar la movilidad estudiantil internacional.

El marco conceptual a partir del cual se construye el análisis se basa en la teoría de la dependencia y el concepto de campo. En la literatura que analiza las relaciones académicas y científicas internacionales, se planteó la cuestión de la dependencia académica y científica de los países periféricos, particularmente en América latina (Beigel, 2010; Kreimer, 2006; Vessuri, 1986; Vessuri et al., 1983). ${ }^{1}$ Beigel define la dependencia académica como "situaciones de dominación

\footnotetext{
${ }^{1}$ Se combina aquí, tal vez de forma abusiva, literatura que trata de la ciencia con literatura que trata de la universidad. Se entiende sin embargo que son cosas diferentes: si bien la universidad produce conocimientos científicos, también los transmite, o sea que tiene una dimensión científica y educativa. Se combina también literatura que trata más específicamente de las ciencias sociales con aquella que trata de las ciencias de forma más general. Si bien existen diferencias, para los fines del presente artículo estas literaturas se pueden asimilar.
} 
que devienen de la posición de un campo académico en el sistema académico mundial"(Beigel, 2010: 15). La configuración de ese "sistema académico mundial" proviene del proceso histórico de división del trabajo científico que se institucionaliza con el proceso colonial y que da lugar, con la descolonización, a una división internacional de la cooperación científica (Shinn et al., 2012). Tal como lo señala Keim, la ciencia en los paises periféricos se caracteriza por ser subdesarrollada en términos materiales, dependiente en términos intelectuales y marginal en términos de reconocimiento internacional (Keim, 2010). ${ }^{2}$ Dentro de las investigaciones sobre las relaciones de dependencia, se prestó particular atención a la movilidad estudiantil en la medida en que permite analizar los mecanismos de circulación de los conocimientos, las relaciones Norte-Sur y la fuga de cerebros (Didou y Gérard, 2009; Didou y Renaud, 2015). El estudio de las movilidades estudiantiles es, según esta literatura, un elemento relevante para el análisis de las relaciones de dependencia académica y científica.

El otro concepto sobre el cual se apoya el análisis es el concepto de campo, utilizado por otros autores para analizar las relaciones universitarias y científicas internacionales (Beigel, 2010; Gingras, 2002; Kauppi y Erkkilä, 201 1; Marginson, 2008). La sociología de Bourdieu dio también lugar a una literatura que le prestó especial atención a la formación, la circulación y la internacionalización de las élites (Attencourt y Garcia, 2015; Dezalay y Garth, 2002; Gérard y Wagner, 2015; Wagner, 2014; Wagner y Réau, 2015).

Por otro lado, la sociología de Bourdieu también está siendo utilizada en relaciones internacionales (Adler-Nissen, 2013). Mérand (2015) propone, por ejemplo, analizar la mundialización como un proceso de formación de campos internacionales. Sapiro señala que el campo no es un concepto nacional sino que es "un concepto abstracto que permite la autonomización metodológica de un espacio de actividad definido de forma relacional (según principios de oposición estructural que dibujan una topografía de posiciones en función de la distribución del capital específico) y dinámica" (Sapiro, 2013). ${ }^{3}$ El concepto de campo se puede por lo tanto utilizar como un instrumento metodológico para construir objetos de investigación internacionales. En los estudios que se apoyaron en la sociología de Bourdieu se suele usar el concepto de "campo transnacional" (Kauppi, 2018; Sapiro, 2018). Esta formulación permite analizar las dinámicas internacionales y sus relaciones con los campos nacionales sin perder de vista la autonomía de los campos nacionales, punto que precisamente enfatiza Gingras cuando analiza las formas específicas de la internacionalidad del campo científico, espacio a la vez nacional e internacional (Gingras, 2002): las ciencias, aunque estén internacionalizadas, siguen ancladas en las naciones (Kleiche, 2018). Se usará por lo tanto en este artículo el concepto de campo universitario transnacional.

$\mathrm{El}$ conjunto de esta literatura conduce a formular el postulado según el cual se puede analizar el campo universitario transnacional y mostrar las relaciones de dependencia entre campos nacionales a partir del estudio de la movilidad estudiantil. Desde ya se entiende que el estudio de la movilidad estudiantil es en sí insuficiente para abarcar la complejidad del funcionamiento del campo universitario transnacional: un análisis exhaustivo debería tomar en cuenta las colaboraciones y las movilidades de docentes y de científicos, los flujos económicos, las traducciones, la citaciones, las jerarquías simbólicas, etcétera. No obstante, aunque sea insuficiente, se sostiene que un estudio de este tipo no deja de ser relevante y permite mostrar parte de la estructura del campo universitario transnacional. La movilidad estudiantil se puede

\footnotetext{
${ }^{2}$ Keim razona explícitamente por analogía con el modelo de dependencia y desarrollo de Cardoso y Faletto (1969).

${ }^{3}$ Traducción propia.
} 
concebir como una manifestación de la relación entre universidades y entre países que resulta de vínculos pasados y actuales, y sienta las bases de vínculos futuros. Se considera en ese sentido que el espacio de la movilidad internacional es un dimensión del campo universitario transnacional y que su análisis permite dar cuenta de los procesos de producción y de reproducción de ese campo, en el sentido de Bourdieu y Passeron (2005).

De manera coherente con el concepto de campo y la sociología de Bourdieu, el estudio se apoya en el Análisis Factorial de las Correspondencias (AFC). En la literatura que se revisó, se pudieron detectar estudios que implementaron otras técnicas estadísticas como la regresión multivariada, el análisis de redes y el escalamiento multidimensional (Perkins y Neumayer, 2014; Rodríguez et al., 2011; Shields, 2013, 2016). El AFG fue también utilizado por Börjesson (2017) para analizar la movilidad estudiantil internacional en 2010. Con el propósito de aportar nuevos elementos al análisis hecho por IESALC y a los estudios que se señalaron, se propone hacer un AFG con los mismos datos que los que utiliza el IESALG, o sea los datos del Instituto de Estadística de la UNESCO para 2017.

El AFG es un método de análisis geométrico de datos que fue particularmente utilizado por Bourdieu (Lebaron y Le Roux, 2015). ${ }^{4}$ Se trata de un método de análisis factorial aplicado al estudio de tablas de contingencia, o sea tablas como las de movilidad, donde en línea se encuentran los países de origen y en columna los países de destino. El AFC permite mostrar la estructura de relaciones entre las líneas y las columnas de una tabla de contingencia midiendo y representando gráficamente a través de la prueba estadística del $\mathrm{Khi}^{2}$ la significatividad de la distancia a la independencia de la relación entre variables (Husson et al., 2016). Aplicado a una tabla de movilidad estudiantil internacional, permite establecer la relación estadística entre países de origen y países de destino, o sea analizar simultaneamente la movilidad saliente y entrante. El AFC es particularmente interesante dado que permite establecer perfiles con base en las similitudes y diferencias que se observan en las relaciones entre líneas y columnas, o sea establecer de manera inductiva una tipología identificando grupos de países de destino que son similares por el origen de sus estudiantes y que se distinguen de otros países que reciben estudiantes de otra procedencia. A la inversa, permite establecer grupos de países que son similares, o que difieren, por el destino de sus estudiantes. De esta forma se establecen las correspondencias en los perfiles de origen y los perfiles de destino, y se realiza la topografía del espacio de la movilidad.

El AFC difiere del uso que se hizo de la regresión multivariada para estudiar la movilidad estudiantil internacional: no se busca elaborar un modelo ceteris paribus de la movilidad a partir de otras variables, sino que se trata de identificar la estructura de la movilidad. El AFG es un método esencialmente inductivo que no implica formular hipótesis a priori y se basa en un razonamiento estructural y relacional (Lebaron y Le Roux, 2015). ${ }^{5}$ Así, se trata de un enfoque de sociología de relaciones internacionales, más empírico-inductivo que hipotético-deductivo: el objetivo es describir, representar e interpretar (Devin, 2013, 2016). El concepto de campo se concibe aquí

\footnotetext{
${ }^{4}$ El Análisis Geométrico de Datos reúne varias técnicas, como el Análisis Factorial de las Correspondencias y el Análisis de las Correspondencias Múltiples. Se llama también Análisis Factorial o, en Francia, análisis de datos.

${ }^{5}$ No se retoman aquí los debates sobre la posibilidad de la inducción pura: el concepto de campo constituye en sí un marco teórico, y la teoría de la dependencia sirve para la interpretación. Sin embargo se enfatiza que el método aplicado no apunta a testear hipótesis ni a elaborar un modelo, como con la regresión multivariada. Tal como lo señalan Lebaron y Le Roux, el objetivo de Benzecri cuando crea el análisis factorial es elaborar un método estadístico que permita identificar una estructura de datos. Uno de los principios claves del análisis factorial es primero describir, luego inferir (Lebaron y Le Roux, 2015).
} 
como un concepto metodológico y el AFC como un instrumento para hacer la descripción, la representación y la interpretación. Por lo tanto, es un enfoque diferente de los otros que se mencionaron, lo cual no impide que sean complementarios y, de hecho, se obtienen resultados convergentes.

El análisis propuesto permitirá establecer una geografia de la movilidad estudiantil internacional, lo cual es un tema fundamental para el análisis de la movilidad (Brooks y Waters, 2011). El AFG permite, a partir del concepto de campo, hacer la topografía de un espacio social, es decir, representar geométricamente un espacio de relaciones. En el caso de este artículo, se propone describir el espacio social de la movilidad estudiantil internacional como dimensión del campo universitario transnacional. $\mathrm{Al}$ usar como variables los países, y al establecer las relaciones entre países de origen y de destino, la descripción del espacio social conduce a establecer el espacio geográfico. Se podra analizar a qué regiones geográficas corresponden los polos que aparecen en el espacio social, lo cual servirá para la interpretación.

El método empleado tiene también numerosos inconvenientes y límites inherentes al uso de la estadística en ciencias sociales, algunos de los cuales conviene señalar brevemente. En primer lugar, se depende de los datos existentes, y la producción de esos datos es en sí un proceso social y político. Los datos son así incompletos: por ejemplo, en la base utilizada no hay datos sobre México como país de destino, y en cuanto a país de origen, sólo hay datos para la movilidad hacia Estados Unidos (EUA) y Canadá. Los datos para los otros países aparecen en la categoría "desconocido", especificándose sólo la región de destino y no el país. La base de datos utilizada implica también tomar como unidad de análisis los Estados: un holandés que va a estudiar a Alemania hace así una movilidad internacional, mientras que un estudiante de Ushuaia que va a Buenos Aires no aparece en los datos de la movilidad internacional, cuando se podría clasificar a Europa como un solo país dada la organización de sus sistema político y universitario. El abordaje es así interestatal, problema que remite a los constructos geográficos que plantea Ferrari-Martínez (2019). Los resultados que se presentan a continuación no son por lo tanto una verdad absoluta y exacta, sino que son un intento para construir una metodología que permita hacer una serie de observaciones sobre un fenómeno complejo y cambiante.

El enfoque propuesto es esencialmente inductivo. Se mostrarán de la forma más rigurosa posible los distintos pasos que se siguieron, particularmente los criterios que se eligieron para hacer las agregaciones que se tuvieron que hacer, teniendo en cuenta que estas agregaciones siempre son discutibles dado que corresponden a tomas de decisiones que se hacen durante el proceso de investigación. Siendo que el análisis en términos de campo es estructural y relacional, se tiene necesariamente que proceder primero a la descripción de la estructura en su conjunto, o sea del espacio social de la movilidad, antes de ubicar y describir la posición de América latina en ese espacio.

\section{El espacio global de los estudiantes internacionales \\ Análisis preliminar}

En este estudio se utilizaron los datos sobre movilidad internacional para 2017 del Instituto de Estadística de la UNESCO, los mismos datos que usó el IESALC (2019) en su estudio. Esa base de datos inicial contiene 213 países de origen y 92 países de destino. ${ }^{6}$ Se procedió primero a un análisis de los principales países de destino y de origen de los estudiantes internacionales. Luego se agregaron las variables país de destino, y después se realizó el AFG de la movilidad global.

\footnotetext{
${ }^{6}$ Se eliminaron de la base de datos los países de destino o origen que no registraban ningún estudiante.
} 


\section{Gráfica 1. Principales países de destino de la movilidad estudiantil internacional (12 primeros) ${ }^{7}$}

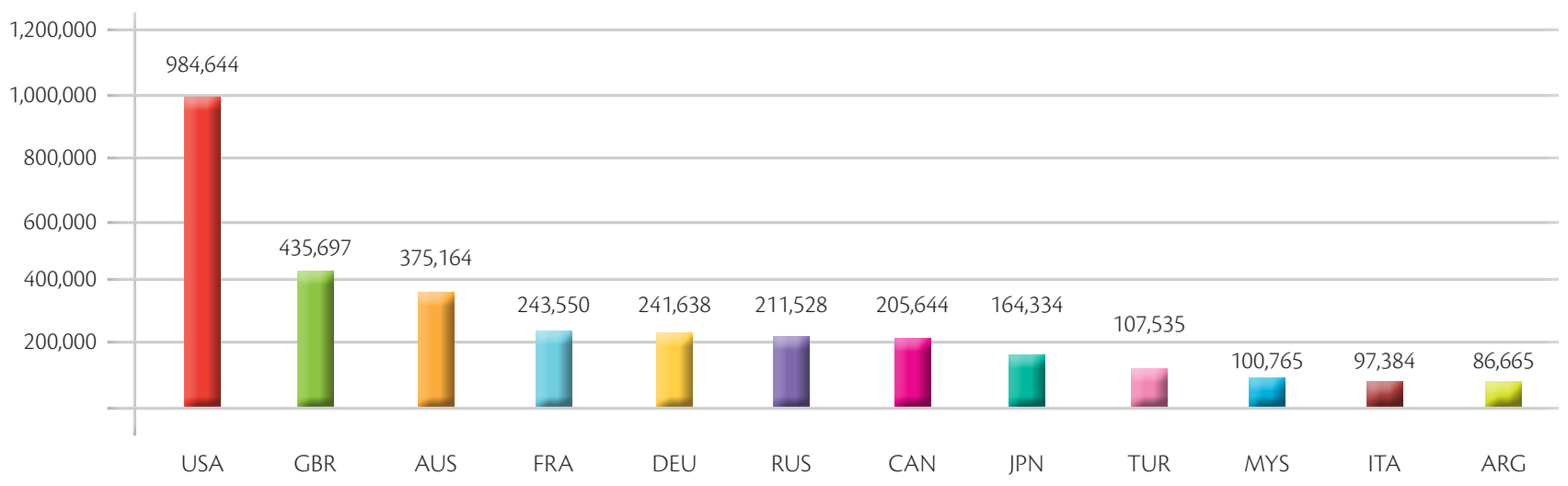

Fuente: elaboración propia con datos del Instituto de Estadística de la unESCO para 2017

La movilidad internacional alcanzaba en 2017 la cantidad de 4637090 estudiantes. Si este número es importante en cantidad, y es el foco de atención de muchos análisis sobre internacionalización, cabe destacar que a lo largo de los últimos 30 años se mantuvo estable alrededor del 2\% de la población estudiantil mundial, y que el incremento cuantitativo de la movilidad se debe esencialmente al crecimiento de la población estudiantil mundial, particularmente al incremento de la población estudiantil china e india (Strassel, 2018). Tal como lo muestra la gráfica 1, el primer país de destino de la movilidad estudiantil internacional es EUA con casi un millón de estudiantes, más del doble que Gran Bretaña, segundo país de destino. Los tres primeros países de destino son anglosajones, y acumulan el $38.7 \%$ de la movilidad internacional. La cuarta y quinta posición son ocupadas por Francia y Alemania. Los cinco primeros países son por lo tanto países occidentales y desarrollados, y acumulan el $49.1 \%$ de la movilidad total, lo cual ya es un indicador de la posición central que ocupan.
Rusia se encuentra en sexta posición, y luego Canadá seguido por varios países asiáticos. El primer país latinoamericano que aparece en esta clasificación es Argentina con un número muy inferior al de EUA y un porcentaje mínimo del total, pero que no deja de ser importante si se tiene en mente el tamaño de su población, sus recursos y su posición en el campo académico transnacional. Con Turquía y Malasia, son los tres únicos países del Sur en esta clasificación.

El estudio de los países de origen permite completar este análisis preliminar. Tal como lo muestra la gráfica 2, el primer país de origen de los estudiantes internacionales es China, seguido por India, los cuales representan el $26.9 \%$ de la movilidad total, porcentaje que indica el peso de estos países en los flujos estudiantiles internacionales. Se puede notar que Francia, Alemania y EUA son también países importantes en cuanto a lugar de origen, y que los países de origen son más variados que los países de destino, dada la presencia de Nigeria, Arabia Saudita y Kazakstán.

\footnotetext{
${ }^{7}$ Para facilitar la lectura de las gráficas y no sobrecargarlas se usa el código país ISO de tres letras, reservando las minúsculas para los países de origen y las mayúsculas para los países de destino. Los números en la gráfica indican la cantidad de estudiantes.
} 


\section{Gráfica 2. Principales países de origen de la movilidad estudiantil internacional (12 primeros)}

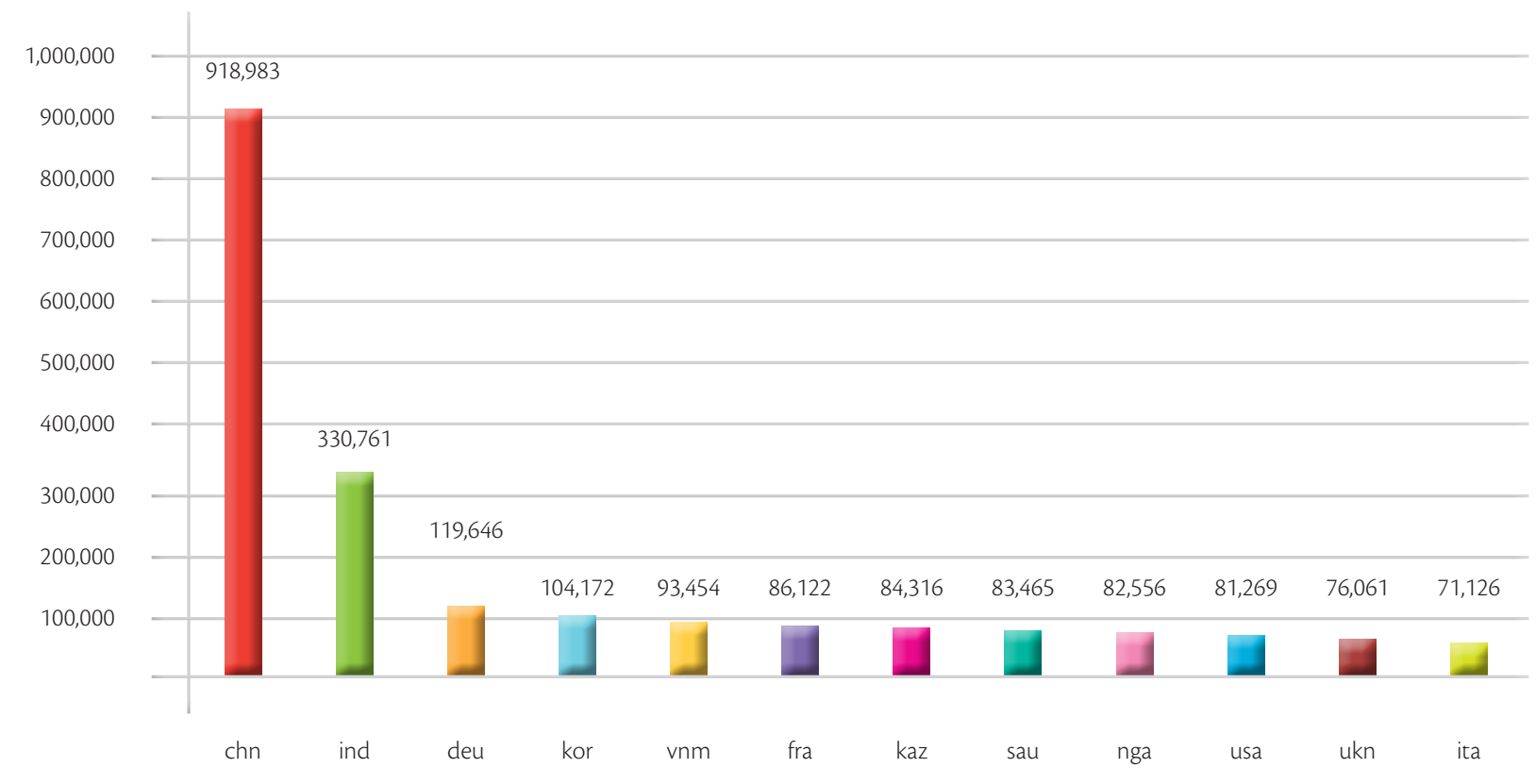

Fuente: elaboración propia con datos del Instituto de Estadística de la UNESCO para 2017.

\section{Agregación y AFC}

Tras este análisis preliminar se procedió a una agregación de las variables país de destino. Dada la diferencia que se observa en la gráfica 1 entre Japón y Turquía, se agregaron las variables cuya cantidad de recepción de estudiantes era inferior a la de Japón. La agregación se hizo en función de criterios geopolíticos (por ejemplo, distinguiendo distintas regiones para Europa), lingüísticos y culturales (por ejemplo, para los países árabes) e institucionales (por ejemplo, el hecho de pertenecer a la Unión Europea). Esa primera agregación permitió reducir el número de variables país de destino a 22. Se realizaron después varios AFC para agregar las variables país de destino que tenían perfiles similares, y cuya agregación era pertinente en función de los criterios anteriores (por ejemplo, agregando Australia y Nueva Zelanda), obteniendo al final una base con 213 países origen (línea) y 16 países o regiones de destino (columnas). Esta estrategia permite conciliar criterios cuantitativos con criterios cualitativos y reducir la cantidad de columnas para hacer una AFG estadísticamente significativa y que se pueda interpretar, evitando también imponer a los datos categorías construidas $a$ priori, como Norte/Sur, Desarrollado/No Desarrollado, etcétera. Con el mismo criterio, no se agregaron las variables país de origen. ${ }^{8}$ En la gráfica 3 se puede visualizar el resultado de la agregación.

\footnotetext{
${ }^{8}$ El lector más familiarizado con el AFC entenderá fácilmente al ver el plan factorial cuáles hubieran tenido que ser las agregaciones de las variables origen para mejorar la representación. Se elige aquí una representación que muestre las distintas posiciones de los países y que sea de lectura más accesible.
} 


\section{Gráfica 3. Cantidad de estudiantes por país o región de destino9}

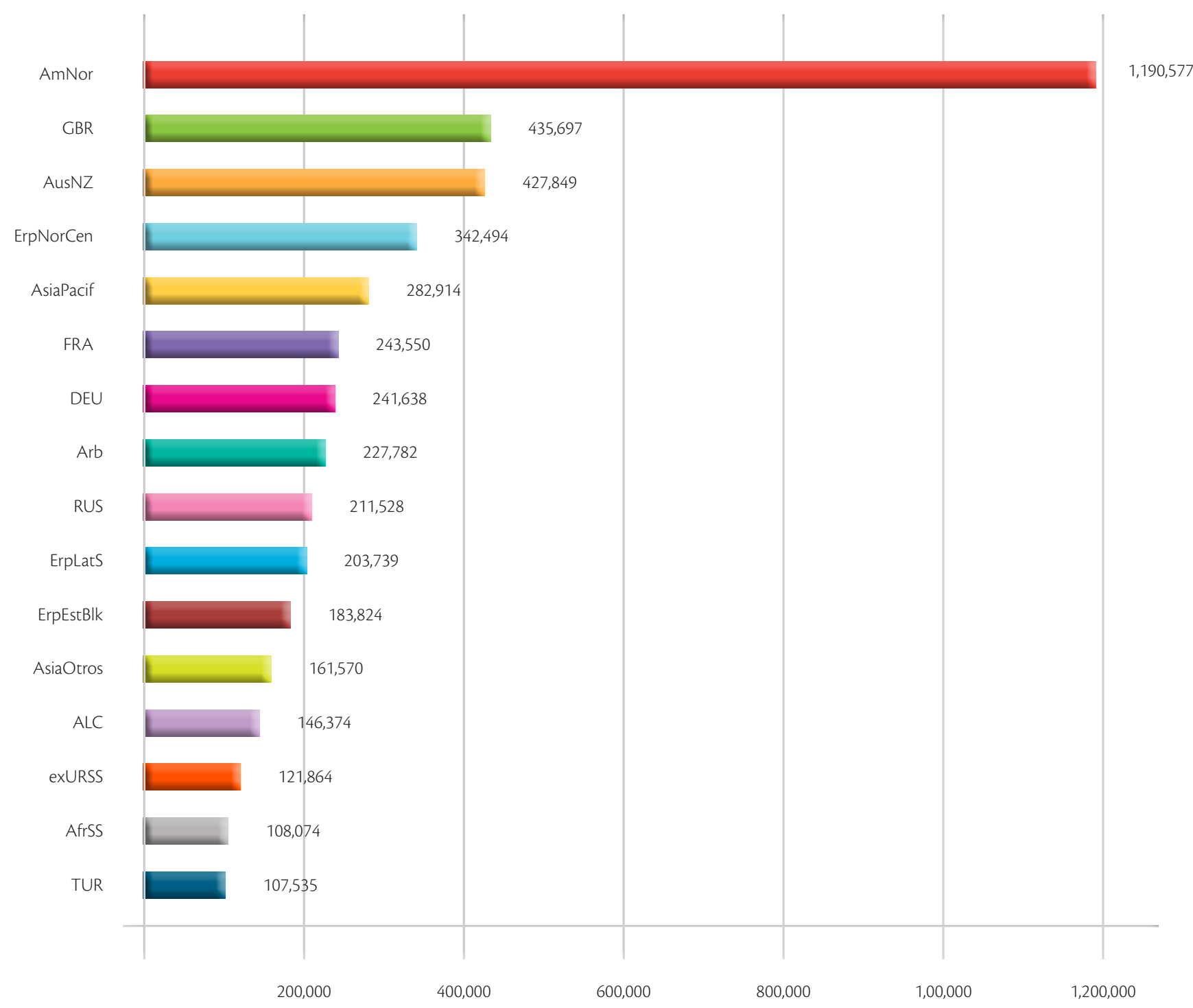

Fuente: elaboración propia con datos del Instituto de Estadística de la unESCO para 2017.

\footnotetext{
${ }^{9}$ América del Norte (AmNor: EUA, Canadá), Australia y Nueva Zelanda (AusNZ), Europa del Norte y Central (ErpNorCen: Andorra, Finlandia, Suiza, Austria, Bélgica, Luxemburgo, Islandia, Dinamarca, Holanda, Irlanda, Noruega, Suecia), Asia Pacífico (AsiaPacif: Japón, Corea, Hong Kong, Macao), países árabes (Arb: Bahréin, Qatar, Omán, Emiratos Árabes Unidos, Arabia Saudita, Marruecos, Túnez, Mauritania, Jordania), Europa Latina y del Sur (ErpLatS: España, Portugal, Italia, Grecia), Europa del Este y Balcanes (ErpEstBlk: Chequia, Estonia, Hungría, Letonia, Lituania, Polonia, Eslovaquia, Eslovenia, Serbia, Bosnia Herzegovina), otros países asiáticos (AsiaOtros: Laos, Vietnam, Indonesia, Brunei, India, Sri Lanka), América Latina (ALG: Chile, Granada, Colombia, República Dominicana, México, Santa Lucía, Brasil, El Salvador, Argentina), ex países soviéticos (ExURSS: Bielorrusia, Ucrania, Georgia, Armenia, Moldavia, Azerbaiyán, Tayikistán, Uzbekistán, Kazakstán, Kirguistán, a los que se sumó Mongolia), África subsahariana (AfrSS: Burundi, Madagascar, Botsuana, Camerún, Ghana, Mozambique, Níger, Ruanda, Cabo Verde, Kenia, Mauricio, Namibia, Benín, Burkina Faso, Costa de Marfil, Senegal, Sudáfrica).
} 
EUA, Canadá, Gran Bretaña, Australia y Nueva Zelanda reciben más de dos millones de estudiantes, lo cual corresponde a casi la mitad de la movilidad estudiantil internacional. China e India representan el $43.1 \%$ de ese total. Esta migración asiática hacia países anglófonos, centrada en el Pacífico, interregional y en un movimiento Sur-Norte, es el elemento fundamental de la estructura de los flujos estudiantiles internacionales. Larsen y Vincent-Lancrin (2002) distinguen dos lógicas de internacionalización de la educación superior: la lógica comercial, donde la educación es concebida como un servicio mercantil, y la lógica cultural, donde los Estados intervienen subvencionando. Los países que se caracterizan por tener una lógica más comercial son precisamente los cinco países mencionados.

\section{Plan factorial}

Se procedió luego al AFC que se muestra en la gráfica 4. Para quienes no estén familiarizados con este tipo de representación, se puede decir que la lectura de la gráfica es, en un primer nivel de lectura, relativamente fácil. Los puntos azules representan los países de destino, y cuanto más cerca están, más se parecen en cuanto al origen de sus estudiantes. Al contrario, si están lejos es que no tienen el mismo perfil. Por ejemplo, Rusia tiene un perfil de estudiantes entrantes más cercano al de los países de la ex URSS o de Turquía que al de América del Norte o América Latina y el Caribe. América del Norte, Australia, Nueva Zelanda y la región Asia Pacífico tienen perfiles de origen de sus estudiantes muy similares, y diferentes de los que van a Rusia o a América Latina. En cuanto a los puntos rojos, la lógica es la misma pero con el origen. Kazakstán y Tayikistán por ejemplo tienen un perfil muy similar, diferente al del Ucrania.

Este primer plan explica el $29.92 \%$ de la varianza total. ${ }^{10}$ Las variables que más contribuyen al primer eje son RUS, ex URSS y AmNor. Las variables que más contribuyen al segundo eje son ALG, Arb y AfrSS. Se pueden distinguir tres polos: el polo central, el polo ruso y el polo latinoamericano; y se puede destacar que la mayoría de los puntos origen se encuentran en el centro de la gráfica y del lado izquierdo. En este polo central se puede notar una separación entre una zona relacionada con AmNor, AusNZ y AsiaPacif; y otra zona relacionada con Europa. El polo central tiene una polarización interna entre la parte anglófona-Pacífica, que tal como se mostró anteriormente, es la más importante y está relacionada con China e India; y la parte europea que se encuentra dilatada entre un polo ErpNorCen orientado hacia el polo anglófono, el polo ErpLatS, orientado hacia ALC, y el polo ErpEstBlk, claramente orientado hacia el polo ruso y exsoviético. Estos resultados son convergentes con el análisis hecho por Shields en el cual usa el escalamiento multidimensional y muestra la oposición que existe entre los flujos de estudiantes en el oeste y en el este de Europa. El polo central está constituido por países desarrollados y en su mayoría occidentales y, teniendo en cuenta los elementos que se destacaron anteriormente, se puede decir que este polo constituye el centro del espacio global de los estudiantes internacionales y que es anglófono. Esta región se caracteriza por una movilidad intrarregional (por ejemplo dentro de Europa) e interregional (por ejemplo entre Europa y América del Norte). Articula movilidades Sur-Norte y Norte-Norte.

Sobre el primer eje, este polo central se distingue del polo ruso. Se puede remarcar la relación entre Rusia como país de destino y Kazakstán, Tayikistán, Uzbekistán, Kirguistán, Turkmenistán y Bielorrusia, y la cercanía entre las variables país o región de destino RUS y ex URSS. Este polo se caracteriza por una fuerte movilidad interna dentro de lo que era el espacio soviético. Tal como lo señala Kuzhabekova

\footnotetext{
${ }^{10}$ Sólo se estudiarán en este artículo los dos primeros ejes.
} 
(2020), el uso de la dicotomía Norte-Sur tiende a invisibilizar los intercambios académicos existentes en Eurasia y en el espacio postsoviético. Estos resultados confirman también el análisis de Chanskseliania (2016) según el cual aunque Rusia pueda ser considerada un país periférico desde los países centrales, sigue siendo un país central para los Estados de la ex Unión Soviética. Los resultados muestran que existe un polo de movilidad postsoviético que se distingue claramente de otros polos. Los estudiantes de esa región van principalmente hacia esa misma región y representan una porción importante de los estudiantes internacionales en esa región, razón por la cual se distingue del polo central. Cabe enfatizar la diferencia entre China, vinculada con el polo central, y los países de Asia Central, claramente más vinculados con Rusia. Aunque difícilmente se puedan utilizar los conceptos de Norte y Sur para calificar estos flujos, éstos se inscriben sin embargo en una historia particular, la de la URSS y del desarrollo de los sistemas universitarios y científicos en los países soviéticos, también marcada por relaciones asimétricas. Si el polo central se caracteriza por su dimensión interregional, el uso del inglés y por estar centrado en el Pacífico, este polo es claramente regional, continental y rusófono.

\section{Gráfica 4. Plan factorial de la movilidad estudiantil mundial (ejes 1 y 2$)^{11}$}

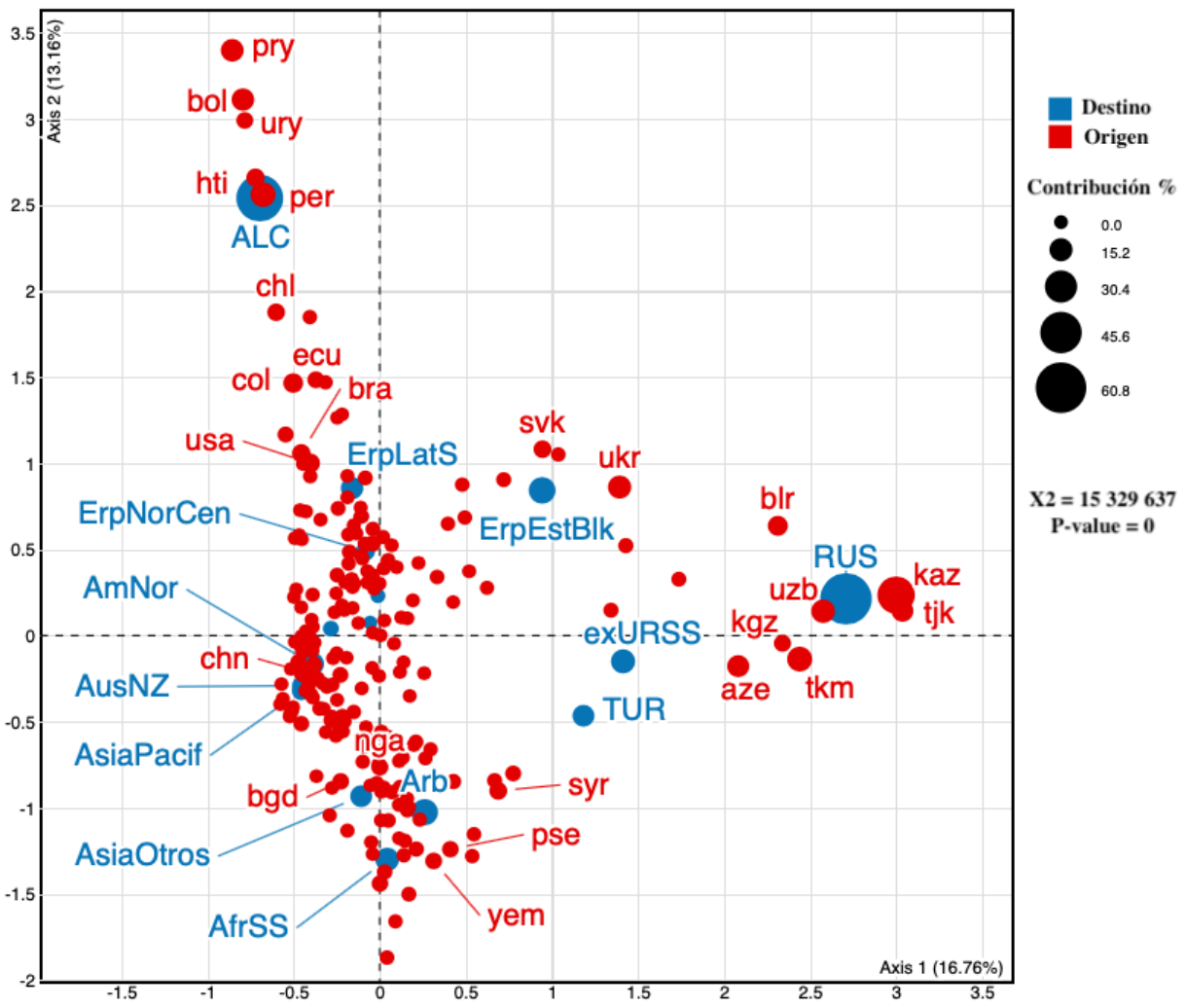

\footnotetext{
${ }^{11}$ Para sintetizar la información se eligió representar en el gráfico las contribuciones. Uno de los elementos que se toman en cuenta en la interpretación de un AFG es la contribución, que indica cuáles son los países que más contribuyen a las diferencias observadas. Los países que mayor contribución tienen son los que permiten interpretar los ejes. Sólo se pusieron las etiquetas de las variables que más contribuyen. Los análisis se realizaron con el paquete estadístico FactoMineR (Husson et al., 2008) y el programa estadístico R y RStudio. El gráfico se realizó con el paquete estadístico explor (Barnier, 2019).
} 
Sobre el segundo eje, se distingue un tercer polo: América Latina. A diferencia de los otros polos, en este caso se trata claramente de una dinámica Sur-Sur y regional. Se puede notar que la variable ErpLatS es la más cercana, lo cual marca una cercanía entre los países latinoamericanos y las antiguas potencias coloniales, España y Portugal particularmente. En su artículo sobre la movilidad estudiantil en el año 2010, Börjesson (2017) señala la existencia de tres polos con lógicas de reclutamiento diferentes y estructuras lingüísticas diferentes: un polo Pacífico, caracterizado por una lógica de mercado y el inglés; un polo Atlántico, franco-ibérico, caracterizado por una lógica colonial y el uso de lenguas latinas (francés, español y portugués) y un polo centro europeo, caracterizado por una lógica de proximidad y el uso de lenguas germánicas y eslavas. Esta estructura parece mantenerse, y se le puede agregar el polo ex soviético, con el ruso.

Estos resultados son convergentes con las investigaciones sobre flujos estudiantiles internacionales hechas por Shields (2013) y Perkins y Neumayer (2014). El modelo de regresión que proponen estos dos últimos autores muestra la importancia de la lengua, de los vínculos coloniales y de la geografía, además de las variables económicas y del prestigio universitario internacional. Estos autores muestran además que dichas variables son más o menos importantes según que se trate de países desarrollados o no, y que existen también diferencias entre los países en desarrollo. El problema del análisis que proponen es que, de forma coherente con la metodología utilizada y la literatura que revisaron, aplican el modelo a grupos de países construidos a priori, por ejemplo, por un lado los países desarrollados y por otro los países en desarrollo. No construyen los grupos de países a partir de una descripción empírica de la estructura de esos flujos. El AFG que se propone aquí permite precisamente clasificar los países a partir del estudio de los flujos, con lo cual sería muy interesante reaplicar el modelo propuesto por estos autores a los grupos que se identifican aquí. Esto permitiría luego fundamentar empíricamente la interpretación que se hace del AFC y analizar más finamente, a partir del modelo, cómo funcionan los distintos polos. Se puede desde ya hacer la hipótesis que las variables lengua y vínculos coloniales son mucho más importantes en el polo América Latina y Rusia, y que las variables económicas y de prestigio universitario son mucho más importantes en el polo anglosajón. El estudio del espacio de la movilidad estudiantil internacional parece en ese sentido indicar que el campo universitario transnacional se estructura con base en la oposición entre un polo central, dominante, anglófono con lógica comercial y de mercado, y polos dominados, ruso y latinoamericano, no anglófonos, con lógicas coloniales y culturales. ${ }^{12}$ Según esta interpretación, Europa se encontraría en una posición intermedia, tensionada por esas distintas lógicas, a la vez que las articula.

Tal como se detallará más adelante, el polo latinoamericano tiene cierta autonomía con respecto al polo central, lo cual lleva a revisar críticamente una visión simplemente "dependentista" de la situación de América Latina, por lo menos en cuanto a la movilidad de los estudiantes. ${ }^{13}$ Si América Latina no está en el centro, y ocupa en ese sentido una posición periférica, el AFC sugiere una fuerte lógica regional de la movilidad, o sea cierta autonomía con respecto al polo central, lo cual contrasta por ejemplo con las dinámicas asiáticas: China está mucho más relacionada con EUA que con Asia y es, sin embargo, una potencia científica y cultural.

\footnotetext{
${ }^{12}$ Se puede formular aquí otra hipótesis: que existe una relación de homología estructural entre los distintos discursos políticos y científicos sobre la internacionalización de la educación superior, o sea las tomas de posiciones, y la posición de los agentes en el campo universitario transnacional.

${ }^{13}$ Se resalta también que un estudio completo de la relación entre dependencia y movilidad debería contemplar otros elementos, como el saldo entre movilidad entrante y saliente así cómo la proporción de estudiantes en movilidad con respecto a la población estudiantil, elementos que el IESALC analiza en su estudio, a los cuales habría que agregar el nivel de estudio, la disciplina y el idioma.
} 
El AFG muestra también que ciertos países, como Brasil, Colombia, Chile y Ecuador tienen más relación con Europa Latina y del Sur, mientras que otros países, como Haití, Perú, Paraguay, Uruguay y Bolivia están más relacionados con América Latina, lo cual sugiere que ciertos países de la región tienen un perfil mucho más regional, en el sentido que tienen una movilidad internacional más intrarregional; mientras que otros países tienen un perfil con mayor movilidad interregional. El objetivo de la siguiente sección es precisamente indagar más en las características de América Latina.

\section{América Latina: ¿dependencia bicéfala o autonomía?}

Se propone en esta última parte prolongar los elementos anteriores detallando el caso de América Latina. En su estudio, el IESALC muestra que la mayoría de los estudiantes de ALC va hacia EUA y Europa. Si este dato, y su significado, son claros, cabe resaltar que la pregunta implícita a la que contesta ese dato es cuál es la relación de la región con los países centrales, o sea con el centro. De ahí que el IESALC haya agregado EUA y Europa. En el análisis que se hizo aquí, se mostró que hay diferencias en la posición de América del Norte y de Europa; y que dentro de Europa hay una suerte de tensión entre una parte de Europa más cercana de América del Norte, otra de América Latina y el Caribe, y otra del espacio ex soviético. Se recalcó también que los países latinoamericanos son más cercanos de Europa Latina y del Sur que de Europa del Norte y Central. Esto parece indicar que, para América Latina, la tensión que se observa dentro del polo central entre los países anglosajones y Europa es particularmente importante.

De manera coherente con estos resultados, y con el propósito de aportar nuevos elementos de análisis, se evaluó el destino de los estudiantes latinoamericanos no en función de su relación con "el centro", sino con "los centros", o sea por un lado los países anglosajones y por otro lado las antiguas potencias coloniales, tal como aparece en la gráfica 5 .

El primer elemento que cabe señalar es que alrededor de un tercio de la movilidad internacional latinoamericana se queda en la región. No se especificó en la gráfica, pero la movilidad hacia América del Norte alcanza 89751 estudiantes, con lo cual los estudiantes latinoamericanos van más hacia la propia región que hacia EUA y Canadá. La movilidad saliente total de América Latina y el Caribe alcanza 309 662, de los cuales 230155 - sumando EUA, Canadá y ALC- hacen una movilidad americana, o sea dentro del continente. Teniendo en cuenta las dimensiones lingüísticas e histórica que se señalaron, resulta interesante constatar que la movilidad hacia países anglófonos es apenas superior a la movilidad hacia la propia región, y que la relación con Europa es principalmente una relación con las antiguas potencias coloniales.

Esto parece confirmar que la movilidad latinoamericana refleja la polarización interna al polo central: por un lado, una fuerte relación con los países anglófonos, y por el otro una relación privilegiada, dentro de Europa, con las antiguas potencias coloniales. La dependencia de la región con el centro es así bicéfala: por un lado, los países anglosajones, y particularmente EUA, potencias actuales, ubicadas del lado Atlántico y Pacífico, relacionadas con la lógica comercial de la internacionalización. Del otro, con menor importancia, las antiguas potencias coloniales, de lenguas latinas, ubicadas del lado Atlántico, relacionadas con la lógica cultural y colonial de la internacionalización. 


\section{Gráfica 5. Movilidad saliente de estudiantes de América Latina y el Caribe hacia otras regiones del mundo (cantidad) ${ }^{14}$}

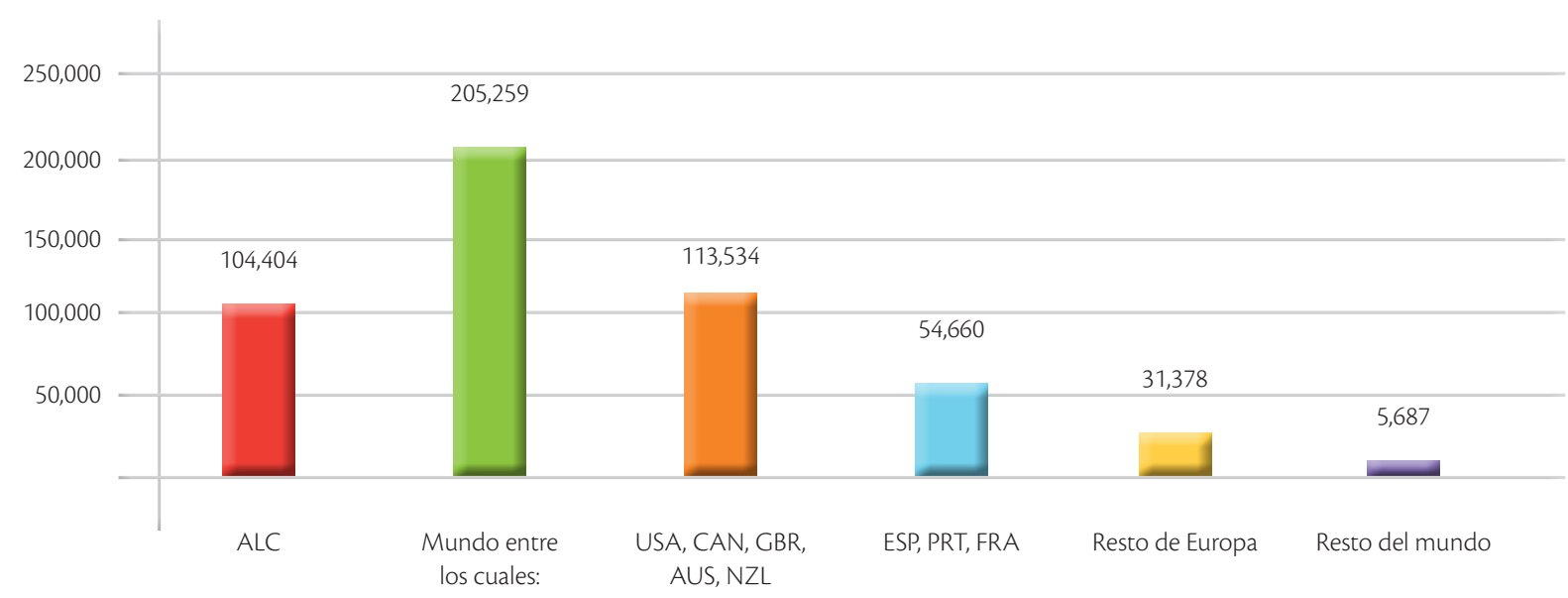

Fuente: elaboración propia con datos del Instituto de Estadística de la UNESCO para 2017.

\section{Gráfica 6. Plan factorial de la movilidad saliente de América Latina y el Caribe (Ejes 1 y 2) ) $^{15}$}

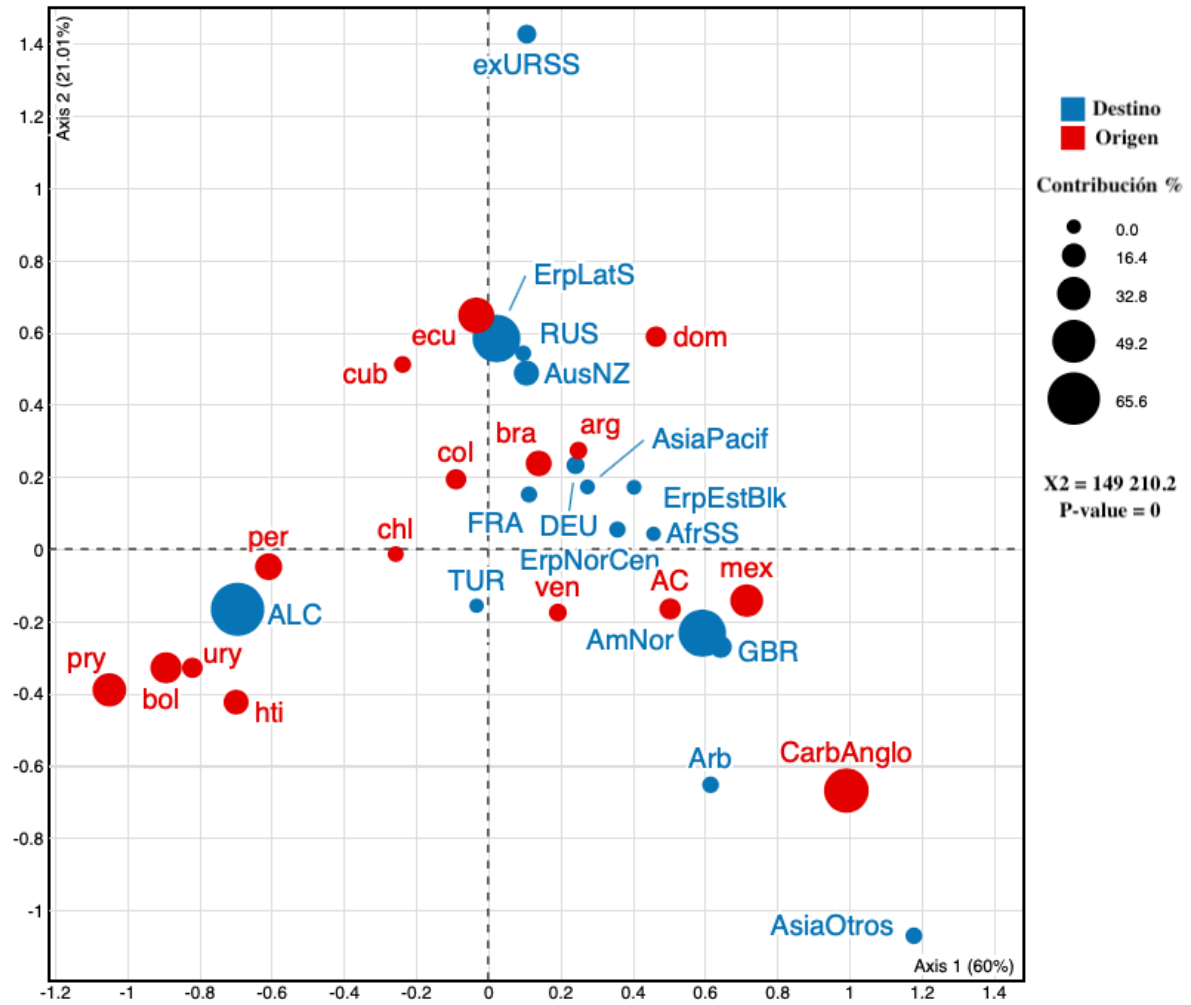

Fuente: elaboración propia con datos del Instituto de Estadística de la UNESCO para 2017.

${ }^{14}$ Pueden notarse diferencias con los datos de IESALC por el hecho de que aquí los países del Caribe quedan incluidos en América Latina, ahí donde el IESALC, dadas las características de esos países con respecto a la región, elige analizarlos aparte.

${ }^{15}$ En este plan factorial se conservaron las mismas variables destino. En cuanto a los países de origen, se agregaron los países de América Central (AC) y los del Caribe anglófono (CarbAnglo, incluyendo a Surinam, Guyana y Belice). 
El plan factorial de la movilidad saliente que se presenta en la gráfica 6 permite mostrar las contradicciones de la tensión entre dependencia bicéfala y autonomía. Ese plan representa el $81 \%$ de la varianza total, con lo cual representa muy bien los diferentes perfiles de la movilidad estudiantil latinoamericana saliente por país. El primer eje, que representa el $60 \%$ de la varianza total, opone claramente América del Norte y América Latina. El segundo eje marca la oposición entre los países más relacionados con Europa Latina y del Sur, y los que están más relacionados con América. Se pueden distinguir tres grupos de países: un primer grupo, con Haití, Bolivia, Paraguay y Perú, con una movilidad regional; un segundo grupo, con México, América Central y el Caribe Anglófono, relacionados con EUA y Gran Bretaña, y un tercer grupo, con
Argentina y Brasil, relacionados con Europa Latina.

En la tabla 1 se detalla aún más el argumento: en el caso del primer grupo, el 71.6\% de los estudiantes internacionales van hacia América Latina, mientras que para el segundo y tercer grupo ese porcentaje es del $6.9 \%$ y del $22.3 \%$. Argentina y Brasil son los que, porcentualmente, más estudiantes mandan hacia Europa Latina y del Sur, con 18.9\%; mientras que México, América Central y el Caribe anglófono mandan casi el 60\% de sus estudiantes hacia América del Norte. La tensión entre dependencia bicéfala y autonomía remite así a una diferencia entre el Norte de la región (México, América Central y el Caribe anglófono) y América del Sur. Esa tensión es particularmente visible en el Caribe: el Caribe anglófono está relacionado con América del Norte, Haití con América Latina y Cuba con Europa Latina y del Sur.

Tabla 1. Porcentaje de estudiantes por grupo de país hacia zona de destino (en porcentaje)

\begin{tabular}{|c|c|c|c|}
\hline & ALC & ErpLatS & AmNor \\
\hline Haití, Bolivia, Paraguay, Uruguay, Perú & 71.6 & 9.6 & 9.7 \\
\hline México, América Central, Caribe Anglófono & 6.9 & 8.8 & 58.8 \\
\hline Argentina, Brasil & 22.3 & 18.9 & 27.1 \\
\hline
\end{tabular}

Fuente: elaboración propia con datos del Instituto de Estadística de la unESCO para 2017.

Estos datos sugieren también, por lo menos para América del Sur, una jerarquía. Si bien la región puede ser considerada periférica, y conviene aquí distinguir la noción de periferia de la de dependencia, dado que la región es un polo con cierta autonomía por lo menos en cuanto a movilidad, parece también existir una diferencia entre los países, como
Argentina y Brasil, ${ }^{16}$ potencias regionales, que tienen un vínculo privilegiado con Europa; y los países que, como Bolivia, Paraguay y Perú — países periféricos dentro de la periferia-, se encuentran más relacionados con las potencias regionales. Según el IESALC (2019: 16), "los países que cuentan con mayores sistemas de educación superior y mejor financiados, que envían la mayoría de sus estudiantes a países

${ }^{16}$ Lee y Sehoole (2015) hablan para el caso de África del Sur "regional hub". Argentina parece tener en América latina un papel similar al de África del Sur en África. 
de otras regiones, son, paradójicamente, los principales receptores de los estudiantes procedentes de los restantes países de América Latina". El caso de Argentina es particularmente interesante: atrae por si sola tantos estudiantes como el resto de la región y, en 2016 , el $92.7 \%{ }^{17}$ de los estudiantes extranjeros en Argentina estaban inscritos en una carrera de pregrado o grado mientras que en 2018 20\% de los estudiantes argentinos en Francia realizaban un doctorado y $42 \%$ una maestría (Campus France, 2019). La paradoja relevada por el IESALC puede así encontrar un elemento de explicación en las jerarquías y asimetrías internas a la región. Explorar más a fondo esta hipótesis implicaría sistematizar el análisis de la relación entre movilidad y nivel de estudio, lo cual va más allá de las posibilidades de este artículo.

De lado de la movilidad entrante, tal como se muestra en la gráfica 7, los estudiantes internacionales en América Latina y el Caribe provienen esencialmente de la región: una parte sustancial de los estudiantes de la región va hacia la propia región, y esos mismos estudiantes representan la mayoría de la movilidad entrante. Es precisamente esta particularidad que el AFC muestra, y que permite calificar a la región como un polo autónomo que se distingue en el espacio global de la movilidad estudiantil.

\section{Gráfica 7. Movilidad entrante en América Latina y el Caribe (cantidades) ${ }^{18}$}

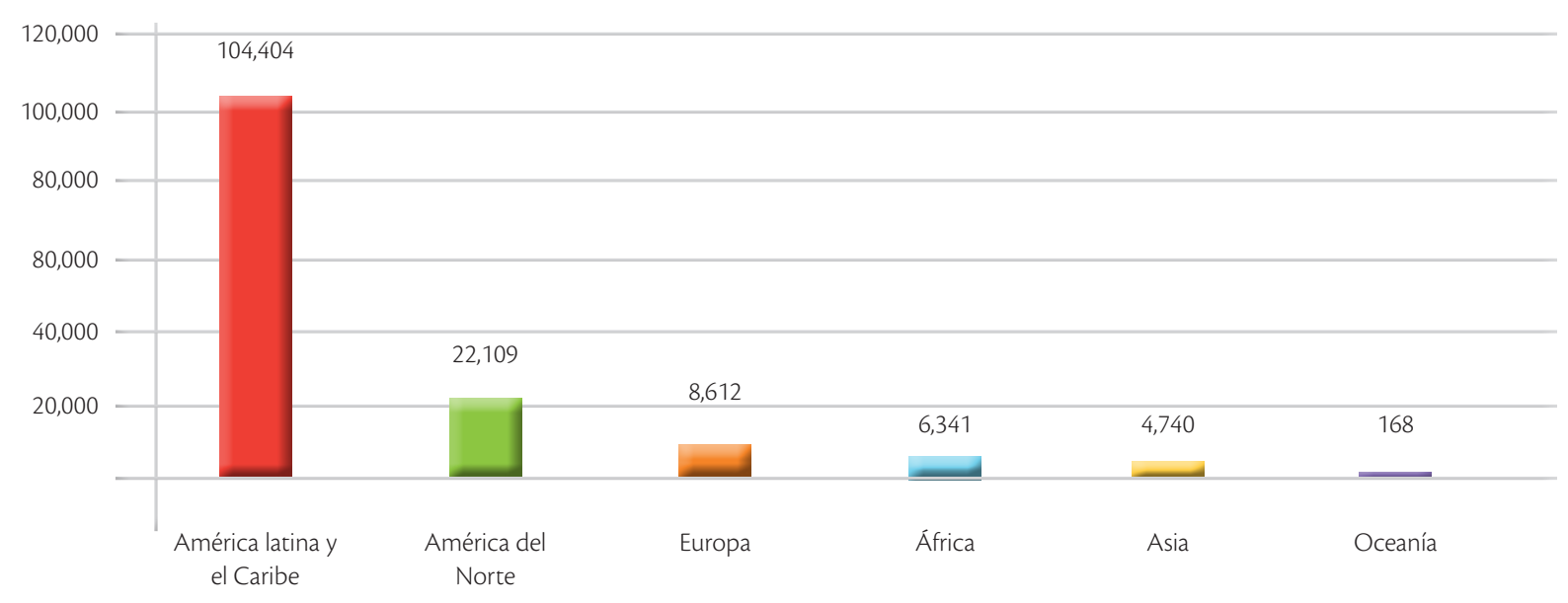

\footnotetext{
${ }^{17}$ Cálculos propios a partir de la base de datos Sistema de Consultas de Estadísticas Universitarias del Ministerio de Educación de Argentina, http://estadisticasuniversitarias.me.gov.ar/.

${ }^{18}$ Las categorías corresponden a las que usa la UNESCO.
} 
Esta gráfica permite también resaltar otros dos elementos: en primer lugar, que existe una movilidad Norte-Sur que alcanza unos 30000 estudiantes, o sea que América Latina atrae estudiantes del Norte, lo cual agrega elementos para considerar que la región es un polo. En segundo lugar, que existe una movilidad Sur-Sur extra regional, particularmente africana, en la cual Brasil tiene un papel clave: de los 6341 estudiantes africanos que van a América Latina, 5120 van hacia Brasil. ${ }^{19}$ Estas movilidades Sur-Sur muestran la existencia de circulación SurSur de conocimientos e indica cambios geopolíticos más amplios (Eyebiyi y Mazzella, 2014). La relativa autonomía en cuanto a movilidad de la región, y el hecho que la región articule una movilidad NorteSur y Sur-Sur extra regional son así otros elementos que permiten caracterizar a la región y discutir de su dimensión periférica y dependiente.

\section{Conclusión}

El estudio realizado permitió mostrar que América Latina constituye un polo dentro del espacio global de los estudiantes internacionales. Aunque periférico, ese polo tiene cierto grado de autonomía, y se encuentra relacionado con el polo central a través de un doble vínculo que refleja una tensión interna al polo central entre los países anglosajones y los países europeos. Esa tensión en el polo central remite a diferencias geopolíticas, lingüísticas e históricas, y refleja también dos lógicas de internacionalización de la educación superior: una lógica comercial y una lógica cultural y colonial. Esa doble vinculación de la región con el polo central, así como el grado de autonomía adquirido, permite distinguir distintos perfiles de países, que remiten a diferencias geopolíticas. México, América Central y el Caribe anglófono se encuentran más vinculados con la parte anglófona del polo central mientras que los países de América del Sur están más vinculados a Europa Latina. A su vez se pudo remarcar que la autonomía observada, y definida en función de la dimensión regional de la movilidad, resulta de la movilidad regional de paises como Haití, Bolivia, Paraguay y Uruguay. La movilidad regional muestra así las ambigüedades de una dependencia bicéfala, y las tensiones entre autonomía y dependencia, dado que la región es también receptora de una movilidad Norte-Sur y Sur-Sur extrarregional.

\footnotetext{
${ }^{19}$ Brasil tiene una política exterior activa en materia educativa y desarrolló programas de cooperación educativa Sur-Sur (Candeas, 2012; Milani et al., 2015; Pinheiro y Beshara, 2012).
} 


\section{Referencias}

Adler-Nissen, Rebecca (2013), Bourdieu in international relations: rethinking key concepts in IR, Nueva York, Routledge.

Attencourt, Boris y Afrânio Garcia (2015), "Compétitions pour la suprématie culturelle et stratégies internationales des élites intellectuelles", en Guide de l'enquête globale en sciences sociales, París, GNRS Éditions, pp. 127-152.

Barnier, Julien (2019), Explor: Interactive Interfaces for Results Exploration, R package version 0.3.5, <https:// GRAN.R-project.org $/$ package $=$ explor $>\quad[$ Consulta: enero de 2020].

Beelen, Jos y Elspeth Jones (2015), "Redefining internationalization at home", en The European Higher Education Area: Between Critical Reflections and Future Policies, Chame, Springer International Publishing, pp. 59-72.

Beigel, Fernanda (2010), Autonomía y dependencia académica. Universidad e investigación científica en un circuito periférico: Chile y Argentina (1950-1980), Buenos Aires, Biblos.

Börjesson, Mikael (2017), "The global space of international students in 2010", Journal of Ethnic and Migration Studies, vol. 43, núm. 8, pp. 1256-1275.

Bourdieu, Pierre y Jean-Claude Passeron (2005), La reproduction: éléments pour une théorie du système d'enseignement, París, Édition de Minuit.

Brooks, Rachel y Johanna Waters (2011), Student mobilities, migration and the internationalization of higher education, Nueva York, Palgrave Macmillan.

Campus France (2019), Fiche Statistique Argentine, Paris, Campus France, <https://ressources.campusfrance. org/publications/mobilite_pays/fr/argentine_fr.pdf $>$ [Consulta: enero de 2020].

Candeas, Alessandro (2012), "Educação e política externa: por uma parceira diplomacia-universidade", en Política externa brasileira: as práticas da política e a política das práticas, Rio de Janeiro, FGV Editora.

Cardoso, Fernando Henrique y Enzo Faletto (1969), Dependencia y desarrollo en América Latina: Ensayo de interpretación sociológica, Buenos Aires, Siglo Veintiuno Editores.
Chankseliani, Maia (2016), "Escaping homelands with limited employment and tertiary education opportunities: outbound student mobility from postSoviet Countries", Population, Space and Place, vol. 22, núm. 3, pp. 301-316.

Devin, Guillaume (2013), Sociologie des relations internationales, París, La Découverte.

Devin, Guillaume (2016), Méthodes de recherche en relations internationales, París, Presses de Sciences Po.

Dezalay, Yves y Bryant Garth (2002), La mondialisation des guerres de palais: la restructuration du pouvoir d'Etat en Amérique Latine, entre notables du droit et Chicago boys, París, Seuil.

Didou Aupetit, Sylvie y Etienne Gérard (2009), Fuga de cerebros, movilidad académica y redes científicas: perspectivas latinoamericanas, México, Cinvestav.

Didou Aupetit, Sylvie y Pascal Renaud (2015), Circulación internacional de los conocimientos: miradas cruzadas sobre la dinámica Norte-Sur, Lima, IRD/IESALC.

Eyebiyi, Elieth y Sylvie Mazzella (2014), "Introduction: Observer les mobilités étudiantes Sud-Sud dans l'internationalisation de l'enseignement supérieur", Cahiers de la recherche sur l'éducation et les savoirs, núm. 14, pp. 7-24.

Ferrari-Martínez, César-Augusto (2019), "Geografías de la movilidad académica internacional: Globalización y discursos de internacionalización”, Revista Iberoamericana de Educación Superior, vol. 10, núm. 29, pp. 180-193.

Gérard, Étienne y Anne-Catherine Wagner (2015), Élites et savoirs, París, Maison de la Recherche et des Sciences de l'Homme.

Gingras, Yves (2002), "Les formes spécifiques de l'internationalité du champ scientifique", Actes de la recherche en sciences sociales, vol. 141-142, núm. 1, pp. 31.

Husson, François, Sébastien Lê y Jérôme Pagès (2016), Analyse de données avec $R$, Rennes, Presses Universitaires de Rennes.

Husson, François et al. (2008), "FactoMineR: An R Package for Multivariate Analysis", fournal of statistical software, vol. 25, núm. 1. 
Institut de Statistique de l'UNESCO (ISU) (2017), Étudiants internationaux dans le pays d'accueil par pays d'origine, année 2017, Institut de Statistique de l'UNESCO, <http:// data.uis.unesco.org> [Consulta: enero de 2020].

Instituto International para la Educación Superior en América Latina y el Caribe (IESALC) (2019), La movilidad en la educación superior en América Latina y el Caribe: retos y oportunidades de un convenio renovado para el reconocimiento de estudios, títulos y diplomas, Caracas, IESALC, UNESCO.

Kauppi, Nikko (2018), "Transnational social fields", en The Oxford Handbook of Pierre Bourdieu, vol. 1, Nueva York, Oxford University Press.

Kauppi, Nikko y Tero Erkkilä (2011), "The struggle over global higher education: actors, institutions, and practices", International Political Sociology, vol. 5, núm. 3, pp. 314-326.

Keim, Wiebke (2010), "Pour un modèle centre-périphérie dans les sciences sociales. Aspects problématiques des relations internationales en sciences sociales", Revue d'anthropologie des connaissances, vol. 4, núm. 3, pp. 570-598.

Kleiche Dray, Mina, (2018), Les ancrages nationaux de la science mondiale: XVIIIe-XXIe siècles, Marseille, IRD.

Knight, Jane (2013), International education hubs, Nueva York, Springer.

Kreimer, Pablo (2006), “¿Dependientes o integrados? La ciencia latinoamericana y la nueva división internacional del trabajo", Nómadas, vol. 24, pp. 199-212.

Kuzhabekova, Aliya (2020) "Invisibilizing Eurasia: How North-South Dichotomization Marginalizes Post-Soviet Scholars in International Research Collaborations", Fournal of Studies in International Education, vol. 1, núm. 24, Ámsterdam, European Association for International Education, pp. 113-130.

Larsen, Kurt; Vincent-Lancrin, Stéphan (2002), "Le commerce international de services d'éducation: est-il bon? est-il méchant?", Politiques et gestion de l'enseignement supérieur, vol. 3, núm. 14, OCDE, pp. 9-50.

Lebaron, Frédéric y Brigitte Le Roux (2015), La méthodologie de Pierre Bourdieu en action, París, Dunod.

Lee, Jenny y Chika Sehoole (2015), "Regional, continental, and global mobility to an emerging economy: the case of South Africa", Higher Education, vol. 70, núm. 5, pp. 827-843.

Marginson, Simon (2008), "Vers une hégémonie de l'université globale", Critique internationale, núm. 39, vol. 2, pp. 87-107.

Mérand, Frédéric (2015), "Bourdieu: le champ, vecteur de la mondialisation", en 10 concepts sociologiques en relations internationales, París, CNRS, pp. 49-70.

Milani, Carlos, Enara Echart Muñoz, Ruben de S. Duarte y Mgno Klein (2015), Atlas de la política exterior brasileña, Buenos Aires, CLACSO.

Perkins, Richard y Eric Neumayer (2014), "Geographies of educational mobilities: exploring the uneven flows of international students", The Geographical fournal, vol. 180, núm. 3, pp. 246-259.

Pinheiro, Leticia y Gregory Beshara (2012), "Politica externa e educação: confluencias e perspectivas no marco da integração regional", en Política externa brasileira: as práticas da política e a política das práticas, Rio de Janeiro, FGV Editora.

Rodríguez González, Carlos, Ricardo Bustillo Mesanza y Petr Mariel (2011), “The determinants of international student mobility flows: an empirical study on the Erasmus programme", Higher Education, vol. 62, núm. 4, pp. 413-430.

Sapiro, Gisèle (2013), "Le champ est-il national?: La théorie de la différenciation sociale au prisme de l'histoire globale", Actes de la recherche en sciences sociales, vol. 200, núm. 5, pp. 70-85.

Sapiro, Gisèle (2018), "Field theory from a transnational perspective", en The Oxford Handbook of Pierre Bourdieu, Nueva York, Oxford University Press, pp. 161-182.

Shields, Robin (2013), "Globalization and international student mobility: a network analysis", Comparative Education Review, vol. 57, núm. 4, pp.609-636.

Shields, Robin (2016), "Reconsidering regionalisation in global higher education: student mobility spaces of the European higher education area", Compare: A Journal of Comparative and International Education, vol. 46, núm. 1, pp. 5-23. 
Shinn, Terry, Dominique Vellard y Roland Waast (2012), "Introduction. La recherche au Nord et au Sud: coopérations et division du travail", Cahiers de la recherche sur l'éducation et les savoirs, vol. 9, pp. 7-31.

Strassel, Christophe (2018), "Les enjeux géopolitiques de la mondialisation universitaire", Hérodote, núm. 168, vol. 1, pp. 9-39.

Vessuri, Hebe (1986), "The universities, scientific research and the national interest in Latin America", Minerva, vol. 24, núm. 1, pp. 1-38.
Vessuri, Hebe, Helena Díaz y Yolanda Texera (1983), La ciencia periférica: ciencia y sociedad en Venezuela, Caracas, Monte Ávila Editores.

Wagner, Anne-Catherine (2014), Les nouvelles élites de la mondialisation: une immigration dorée en France, París, Presses Universitaires de France.

Wagner, Anne-Catherine y Bertrand Réau (2015), "Le capital international: Un outil d'analyse de la reconfiguration des rapports de domination", en Guide de l'enquête globale en sciences sociales, París, CNRS.

\section{Cómo citar este artículo:}

Montoya, Víctor (2021), “¿Dependencia bicéfala o autonomía? América Latina en el espacio de la movilidad estudiantil internacional", Revista Iberoamericana de Educación Superior (RIES), vol. XII, núm. 34, pp. 156-175, DoI: https://doi.org/10.22201/ iisue.20072872e.2021.34.985 [Consulta: fecha de última consulta]. 